\title{
Characteristics Analysis of the Square Laminated Core under DC- biased Magnetization by the Fixed-point Harmonic-balanced FEM
}

\author{
Xiaojun Zhao ${ }^{1}$, Lin $\mathrm{Li}^{1}$, Member IEEE, Junwei Lu², Senior Member IEEE, Zhiguang Cheng ${ }^{3}$, Senior Member IEEE \\ and Tiebing $\mathrm{Lu}^{1}$ \\ ${ }^{1}$ North China Electric Power University, Baoding, 071003, P.R. China \\ ${ }^{2}$ Griffith University, Brisbane, QLD 4111 Australia \\ ${ }^{3}$ R\&D Center of Baoding Tianwei Group Co., LTD, Baoding, 071056, P.R. China
}

\begin{abstract}
The ferromagnetic core of power transformer is susceptible to DC bias and has special nonlinear and hysteretic characteristics under DC-biased magnetization. The DC-biasing magnetization curve of a square laminated core (SLC) can be obtained by DC bias test. The harmonic-balanced finite element method (HBFEM) combined with the fixed-point technique is used to calculate the DCbiasing magnetic field in the SLC based on the measured DC-biasing magnetization curve. A locally convergent method in harmonic domain is presented to determine the fixed-point reluctivity, which can speed up the convergence of harmonic solution. Computations of the DC-biasing magnetic field are performed by means of the basic and DC-biasing magnetization curves respectively. Comparison between calculation and measurement indicates the validity and superiority of the DC-biasing magnetization curve
\end{abstract}

Index Terms-DC-biased magnetization, fixed-point reluctivity, harmonic-balanced FEM, locally convergent method.

\section{INTRODUCTION}

$\mathrm{D}^{\mathrm{c}}$ $\mathrm{C}$ flux and $\mathrm{AC}$ flux coexist in the ferromagnetic core when the power transformer works under DC-biased magnetization which leads to the distortion and asymmetry of hysteresis loops. The loss curve of iron core under DC-biased excitation is also different from that under sinusoidal excitation [1]-[2]. Fig.1 depicts two different hysteresis loops under DC-biased and sinusoidal excitations. $B_{\mathrm{acm}}$ and $B_{\mathrm{dc}}$ are the magnitudes of AC component and the DC component of flux density respectively. The hysteresis loop is distorted seriously and the ferromagnetic core is saturated significantly by the DC flux density $B_{\mathrm{dc}}$. As a result, the magnetization curve under DC-biased excitation is different from that under sinusoidal excitation.

The fixed-point technique has been used in finite element analysis of nonlinear magnetic field under sinusoidal excitation [3], and the optimal convergence strategy is discussed in nonlinear eddy current problems [4]-[5]. Two different methods used there to determine the fixed-point reluctivity are presented in time-stepping FEM and the convergent performance is discussed in detail [6]-[7]. In this paper, the fixed-point technique is combined with the HBFEM to calculate the DC-biasing magnetic field by means of the measured magnetization curves of SLC under DC-biased magnetization, neglecting the hysteretic effect and anisotropic property.

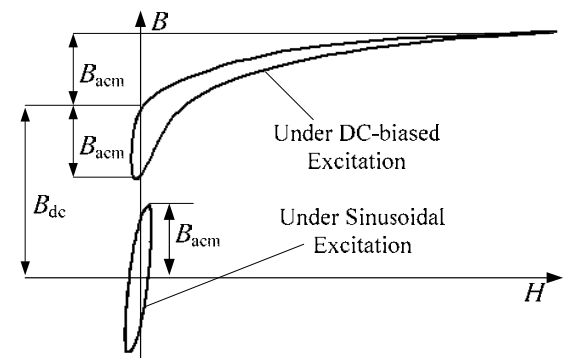

Manuscript received July 1, 2011. Corresponding author: Xiaojun Zhao (e-mail: 158748295@163.com).

Digital Object Identifier inserted by IEEE

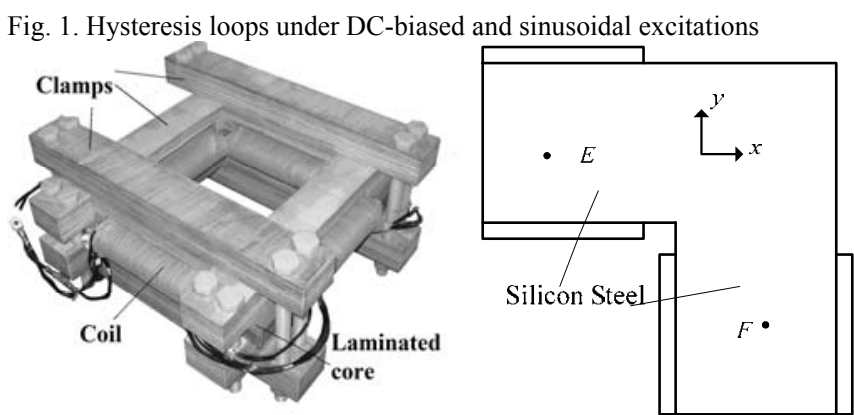

Fig. 2. The SLC and computational region

\section{DC-Biasing Magnetization CuRVE}

The magnetization curve can be treated as the locus of vertices of hysteresis loops. Therefore the hysteresis loops are required to obtain the magnetization curve under DC-biased magnetization. The DC-biasing experiment was carried out on the SLC shown in Fig. 2. The iron core is made up of grainoriented silicon steel (30Q140) and entwined by two coils of both 312 turns. The exciting coil is fed by alternating voltage $(50 \mathrm{~Hz})$ and used for measurement of no-load exciting current $I$, while the search coil is used for measurement of the no-load induced voltage $U_{\mathrm{ac}}$. The DC bias current $I_{\mathrm{dc}}$ is applied to the exciting coil by a direct current source. To obtain the DCbiasing hysteresis loops, the measured data are manipulated by,

$$
\begin{gathered}
\varphi_{\mathrm{ac}}=\left(1 / N_{\text {coil }}\right) \int U_{\mathrm{ac}} d t \\
B=\left(\varphi_{\mathrm{ac}}+\varphi_{\mathrm{dc}}\right) / S \\
H=N_{\text {coil }} I / L
\end{gathered}
$$

where $\varphi_{\mathrm{ac}}$ and $\varphi_{\mathrm{dc}}$ are the AC and DC component of the total flux respectively in the magnetic core, $S$ is the cross-sectional area of the core, and $L$ is the mean length of the magnetic circuit. Since the DC flux $\varphi_{\mathrm{dc}}$ cannot be directly measured experimentally, an iterative method has been proposed in [8] to calculate $\varphi_{\mathrm{dc}}$ : First, $\varphi_{\mathrm{ac}}$ is calculated by (1) and exciting current $I$ can be captured by search coil under a given DCbiasing working condition. An initial value of $\varphi_{\mathrm{dc}}$ is assumed, thus based on the basic magnetization curve and the total flux 
$\varphi$ (sum of $\varphi_{\mathrm{ac}}$ and $\varphi_{\mathrm{dc}}$ ), the calculated exciting current $I_{\mathrm{c}}$ is compared with $I$. Finally $\varphi_{\mathrm{dc}}$ is adjusted iteratively until $I_{\mathrm{c}}$ and $I$ are matched.

$B$ and $H$ can be determined by solving (1)-(3) with the calculated $\varphi_{\mathrm{dc}}$ and a series of hysteresis loops under DC-biased magnetization shown in Fig.3 can be obtained. Consequently the DC-biased $B_{\mathrm{m}}-H_{\mathrm{b}}$ curve [9] can be obtained. Table I presents the measured data, which shows the asymmetry of the DC-biasing magnetization curve. The DC component of magnetic intensity $H_{\mathrm{dc}}$ can be calculated by (3) with the given direct current $I_{\mathrm{dc}}$ in the exciting coil.

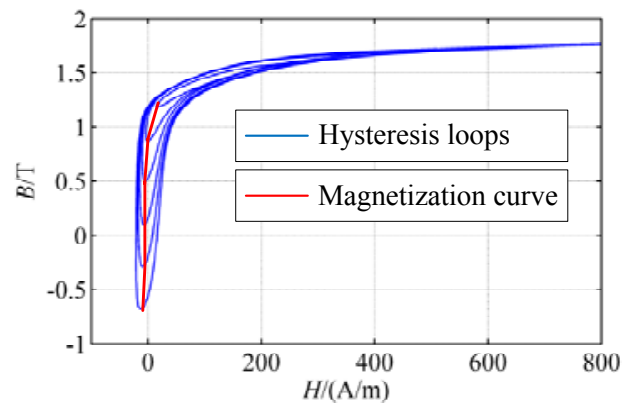

Fig. 3. Measured hysteresis loops under DC-biased magnetization $\left(H_{\mathrm{dc}}=107 \mathrm{~A} / \mathrm{m}\right)$

TABLE I

DATA OF MAGNETIZATION CURVE UNDER DC-BIASED MAGNETIZATION $\left(H_{\mathrm{dc}}=107 \mathrm{~A} / \mathrm{m}\right)$

\begin{tabular}{|c|c|c|c|c|c|c|}
\hline$B_{\mathrm{m}}(\mathrm{T})$ & -1.543 & -1.244 & -0.673 & -0.276 & -0.079 & 0.114 \\
\hline$H_{\mathrm{b}}(\mathrm{A} / \mathrm{m})$ & -104.77 & -32.89 & -13.98 & -10.14 & -8.328 & -7.238 \\
\hline$B_{\mathrm{m}}(\mathrm{T})$ & 0.314 & 0.876 & 1.330 & 1.647 & 1.783 & 1.908 \\
\hline$H_{\mathrm{b}}(\mathrm{A} / \mathrm{m})$ & -6.323 & -0.747 & 47.177 & 325.51 & 1319.6 & 2665.8 \\
\hline
\end{tabular}

\section{FIXED-POINT HARMONIC-BALANCED METHOD}

A relationship between magnetic intensity and flux density is represented by introducing the fixed-point reluctivity $v_{\mathrm{FP}}$ [10],

$$
\boldsymbol{H}(\boldsymbol{B})=v_{\mathrm{FP}} \boldsymbol{B}-\boldsymbol{M}(\boldsymbol{B})
$$

where $v_{\mathrm{FP}}$ is a constant value. $\boldsymbol{M}$ is a magnetization-like quantity which varies nonlinearly with the flux density $\boldsymbol{B}$. Therefore the magnetic intensity $\boldsymbol{H}$ is divided into two parts, the linear part related to $v_{\mathrm{FP}}$ and nonlinear part related to $\boldsymbol{M}$.

The vector potential equation is used to describe the twodimensional nonlinear magnetic field by substituting (4) into the Maxwell's equations,

$$
\nabla \times v_{\mathrm{FP}}(\nabla \times A)+\sigma \frac{\partial A}{\partial t}=J-\nabla \times \boldsymbol{M}
$$

where $A$ is the magnetic vector potential and $\sigma$ is the electric conductivity.

The fixed-point reluctivity determines the convergence of solutions. If $v_{\mathrm{FP}}$ is set as a constant, the fixed-point iteration can be very slow and even not stable especially when $v_{\mathrm{FP}}$ is not selected properly. Actually $v_{\mathrm{FP}}$ can be updated in each iterative step and a fast convergence can be achieved based on the locally convergent method. The local fixed-point reluctivity [6]-[7] can be determined by,

$$
\nu_{\mathrm{FP}}=\left(\partial H_{x} / \partial B_{x}+\partial H_{y} / \partial B_{y}\right) / 2
$$

The periodic variables in the electromagnetic field under DC-biased excitation can be approximated by the Fourierseries with a finite number of harmonics [11],

$$
W(t)=W_{0}+\sum_{n=1}^{\infty}\left(W_{2 n-1} \sin n \omega t+W_{2 n} \cos n \omega t\right)
$$

where $W(\mathrm{t})$ can be replaced by current density $\boldsymbol{J}$, vector potential $A$, flux density $\boldsymbol{B}$, magnetic intensity $\boldsymbol{H}$, and $\boldsymbol{M}$. Equation (4) can be rewritten in isotropic material by means of harmonic vector in harmonic domain,

$$
\left\{\begin{array}{l}
\boldsymbol{H}_{x}=v_{\mathrm{FP}} \boldsymbol{B}_{x}-\boldsymbol{M}_{x} \\
\boldsymbol{H}_{y}=v_{\mathrm{FP}} \boldsymbol{B}_{y}-\boldsymbol{M}_{y}
\end{array}\right.
$$

in which

$$
\left\{\begin{array}{l}
\boldsymbol{H}_{x}=\left[\begin{array}{llllll}
H_{x, 0} & H_{x, 1} & H_{x, 2} & H_{x, 3} & H_{x, 4} & \cdots
\end{array}\right] \\
\boldsymbol{H}_{y}=\left[\begin{array}{llllll}
H_{y, 0} & H_{y, 1} & H_{y, 2} & H_{y, 3} & H_{y, 4} & \cdots
\end{array}\right]
\end{array}\right.
$$

Each of harmonic vectors $\boldsymbol{B}_{\mathrm{x}}, \boldsymbol{B}_{\mathrm{y}}, \boldsymbol{M}_{\mathrm{x}}$ and $\boldsymbol{M}_{\mathrm{y}}$ has a similar form with (9). The fixed-point reluctivity can also be expressed in harmonic domain,

$$
v_{\mathrm{FP}}(t)=d H(B) / d B=v_{0}+\sum_{n=1}^{\infty}\left\{v_{2 n-1} \sin (n \omega t)+v_{2 n} \cos (n \omega t)\right\}
$$

where $v_{2 \mathrm{n}-1}$ and $v_{2 \mathrm{n}}$ are the harmonic coefficients of the fixedpoint reluctivity, while $v_{0}$ is the DC component. The fixedpoint harmonic-balanced equation can be obtained by substituting (7) and (10) into the weak form of (5) based on Galerkin's method:

$$
\begin{gathered}
\boldsymbol{S}_{\mathrm{e}} \boldsymbol{A}_{\mathrm{e}}+\boldsymbol{T}_{\mathrm{e}} \boldsymbol{A}_{\mathrm{e}}-\boldsymbol{K}_{\mathrm{e}}-\boldsymbol{P}_{\mathrm{e}}=\left[\begin{array}{lll}
S_{11} \boldsymbol{D}_{\mathrm{FP}} & S_{12} \boldsymbol{D}_{\mathrm{FP}} & S_{13} \boldsymbol{D}_{\mathrm{FP}} \\
S_{21} \boldsymbol{D}_{\mathrm{FP}} & S_{22} \boldsymbol{D}_{\mathrm{FP}} & S_{23} \boldsymbol{D}_{\mathrm{FP}} \\
S_{31} \boldsymbol{D}_{\mathrm{FP}} & S_{32} \boldsymbol{D}_{\mathrm{FP}} & S_{33} \boldsymbol{D}_{\mathrm{FP}}
\end{array}\right] \cdot\left[\begin{array}{c}
\boldsymbol{A}_{\mathrm{e} 1} \\
\boldsymbol{A}_{\mathrm{e} 2} \\
\boldsymbol{A}_{\mathrm{e} 3}
\end{array}\right] \\
+\left[\begin{array}{ccc}
T_{11} \boldsymbol{N} & T_{12} \boldsymbol{N} & T_{13} \boldsymbol{N} \\
T_{21} \boldsymbol{N} & T_{22} \boldsymbol{N} & T_{23} \boldsymbol{N} \\
T_{31} \boldsymbol{N} & T_{32} \boldsymbol{N} & T_{33} \boldsymbol{N}
\end{array}\right]\left[\begin{array}{c}
\boldsymbol{A}_{\mathrm{e} 1} \\
\boldsymbol{A}_{\mathrm{e} 2} \\
\boldsymbol{A}_{\mathrm{e} 3}
\end{array}\right]-\left[\begin{array}{l}
\boldsymbol{K}_{\mathrm{e} 1} \\
\boldsymbol{K}_{\mathrm{e} 2} \\
\boldsymbol{K}_{\mathrm{e} 3}
\end{array}\right]-\left[\begin{array}{c}
\boldsymbol{P}_{\mathrm{e} 1} \\
\boldsymbol{P}_{\mathrm{e} 2} \\
\boldsymbol{P}_{\mathrm{e} 3}
\end{array}\right]=\mathbf{0}(11)
\end{gathered}
$$

where $S_{i, j}=\int_{\Omega_{e}} \nabla N_{i} \cdot \nabla N_{j} d \Omega, T_{i, j}=\int_{\Omega_{e}} \sigma N_{i} \cdot N_{j} d \Omega$, and the subscripts $i$ and $j$ represent the node number in finite elements, $N_{i}$ is the shape function on node $i$ in finite element region. $\boldsymbol{A}$ is the harmonic form of magnetic vector potential, and $N$ is related to harmonic number and called harmonic matrix [1112]. $\boldsymbol{D}_{\mathrm{FP}}$ is relevant to the reluctivity and can be called fixedpoint reluctivity matrix,

$$
\begin{aligned}
& \boldsymbol{D}_{\mathrm{FP}}= \\
& {\left[\begin{array}{cccccc}
2 v_{0} & v_{1} & v_{2} & v_{3} & v_{4} & \cdots \\
2 v_{1} & 2 v_{0}-v_{4} & v_{3} & v_{2}-v_{6} & -v_{1}+v_{5} & \cdots \\
2 v_{2} & & 2 v_{0}+v_{4} & v_{1}+v_{5} & v_{2}+v_{6} & \cdots \\
2 v_{3} & & & 2 v_{0}-v_{8} & v_{7} & \cdots \\
2 v_{4} & & \text { Symmetry } & & 2 v_{0}+v_{8} & \cdots \\
\cdots & & & & & \ddots
\end{array}\right]}
\end{aligned}
$$

$\boldsymbol{P}$ is derived from magnetization-like quantity $\boldsymbol{M}$,

$$
\boldsymbol{P}_{\mathrm{e} i}=\left[\begin{array}{llllll}
P_{\mathrm{e} i, 0} & P_{\mathrm{e} i, 1} & P_{\mathrm{e} i, 2} & P_{\mathrm{e} i, 3} & P_{\mathrm{e} i, 4} & \cdots
\end{array}\right]^{\mathrm{T}}
$$




$$
P_{\mathrm{e} i, m}=\iint_{\Omega_{\mathrm{e}}}\left(M_{y, m} \frac{\partial N_{\mathrm{e} i}}{\partial x}-M_{x, m} \frac{\partial N_{\mathrm{e} i}}{\partial y}\right) \mathrm{d} x \mathrm{~d} y
$$

The electromagnetic coupling can be considered by,

$$
\boldsymbol{U}_{\text {ink }}=\boldsymbol{C}_{k} \boldsymbol{A}+\boldsymbol{Z}_{k} \boldsymbol{J}_{k}
$$

where the subscript $k$ means the number of external circuit, $\boldsymbol{U}_{\text {in }}$ and $\boldsymbol{J}$ are the harmonic vectors of external input voltage and current density, $\boldsymbol{C}$ and $\boldsymbol{Z}$ represent the coupling matrix and impedance matrix respectively [12].

The magnetic field and exciting current can be calculated by solving (11) and (15) together.

$$
\left[\begin{array}{ccccc}
\boldsymbol{H} & -\boldsymbol{G}_{1} & \cdots & -\boldsymbol{G}_{k} & \cdots \\
\boldsymbol{C}_{1} & \boldsymbol{Z}_{1} & \mathbf{0} & \mathbf{0} & \cdots \\
\vdots & \vdots & \ddots & \mathbf{0} & \cdots \\
\boldsymbol{C}_{k} & \mathbf{0} & \mathbf{0} & \boldsymbol{Z}_{k} & \cdots \\
\vdots & \vdots & \vdots & \vdots & \ddots
\end{array}\right]\left[\begin{array}{c}
\boldsymbol{A} \\
\boldsymbol{J}_{1} \\
\vdots \\
\boldsymbol{J}_{k} \\
\vdots
\end{array}\right]=\left[\begin{array}{c}
\boldsymbol{P} \\
\boldsymbol{U}_{\mathrm{in} 1} \\
\vdots \\
\boldsymbol{U}_{\mathrm{in} k} \\
\vdots
\end{array}\right]
$$

where $\boldsymbol{H}=\boldsymbol{S}+\boldsymbol{T}$, and $\boldsymbol{G}$ is related to spatial distribution of $\boldsymbol{J}$.

The harmonic vector $\boldsymbol{P}$ nonlinearly depends on $\boldsymbol{B}$ and hence on $\boldsymbol{A}$. Therefore, equation (16) can be solved iteratively. In the first step $(p=0)$, initial value are assumed to the vector $\boldsymbol{P}\left(=\boldsymbol{P}_{0}\right)$, $\boldsymbol{A}_{1}$ and $\boldsymbol{J}_{1}$ are computed with the given working voltage $\boldsymbol{U}_{\text {in }}, \boldsymbol{B}_{1}$ and $\boldsymbol{H}_{1}$ can be obtained from $\boldsymbol{A}_{1}$ and the single-valued function $H=F(B)$, which should be Lipschitz continuous [7]. Consequently, the vector $\boldsymbol{P}$ can be updated by $\boldsymbol{M}$ and the computational process is performed iteratively. The stopping criterion is defined by:

$$
\left\|\boldsymbol{X}^{p+1}-\boldsymbol{X}^{p}\right\| /\left\|\boldsymbol{X}^{p}\right\|<\varepsilon
$$

where $\boldsymbol{X}$ represents all harmonic solutions, including $\boldsymbol{A}$ and $\boldsymbol{J}$.

\section{VALIDATION AND DISCUSSION}

Due to the structural symmetry of the SLC, 1922 triangular elements and 1014 nodes are used in a quarter of the model shown in Fig.2 to carry out the harmonic computation.

\section{A. Computational Results and Analysis of the Fixed-Point} Harmonic-Balanced Method

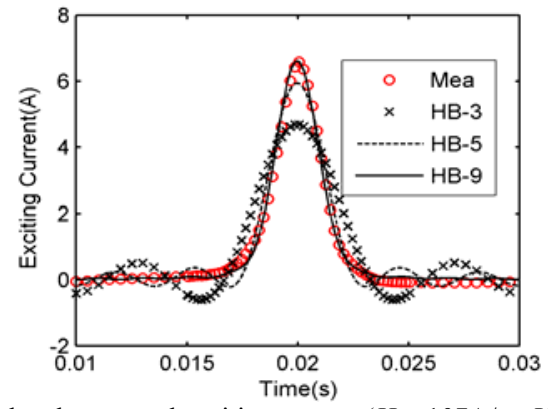

Fig. 4. Calculated and measured exciting current $\left(H_{\mathrm{dc}}=107 \mathrm{~A} / \mathrm{m}, U_{\mathrm{ac}}=260 \mathrm{~V}\right)$

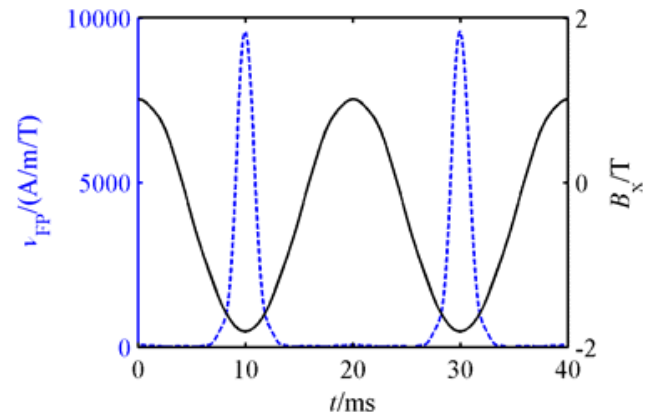

Fig. 5. Waveforms of calculated $v_{\mathrm{FP}}$ and flux density in point $E$ in the laminated core $\left(H_{\mathrm{dc}}=107 \mathrm{~A} / \mathrm{m}, U_{\mathrm{ac}}=260 \mathrm{~V}\right)$

The DC-biasing magnetization curves shown in Table I is employed to calculate the DC-biasing magnetic field by the fixed-point HBFEM. Different harmonics are truncated to calculate the exciting current, which is compared with the measured one in Fig.4. Fig.5 indicates the effectiveness of the locally convergent method in adapting the fixed-point reluctivity according to the changes of the flux density. The waveform of flux density is raised under DC-biased excitation. The negative peak value approaches to -2.0 Tesla, which leads to the significant saturation of the ferromagnetic core.

The convergent speed of harmonic solutions in HBFEM depends on the way to update magnetic reluctivity. The conventional method [12] adopts relaxation iterative scheme to renew the reluctivity matrix, thus the harmonic solutions converge slowly. In the fixed-point harmonic-balanced method determination of $v_{\mathrm{FP}}$ plays an important role in computation. The locally convergent method determines an optimal value of $v_{\mathrm{FP}}$ in a local region, which speed up the convergence of harmonic solution significantly.

Eleven harmonics $\left(N_{\mathrm{c}}=11\right)$ are truncated in both methods to observe the evolution of relative error of harmonic solution in (17). As shown in Fig. 6, the harmonic solution converges fast in the proposed method, but slowly in the conventional method. It is concluded that solutions in the conventional method requires more iterations to reach convergence.

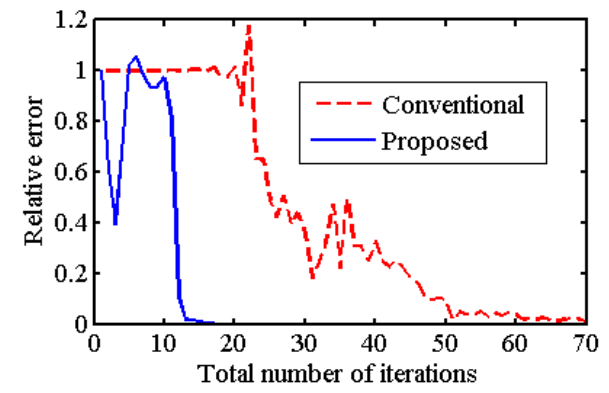

Fig. 6. Evolution of the relative error of harmonic solution in two methods $\left(H_{\mathrm{dc}}=107 \mathrm{~A} / \mathrm{m}, U_{\mathrm{ac}}=370 \mathrm{~V}, N_{\mathrm{c}}=11\right)$

The two methods are compared in detail in Table II. With the same memory requirement, the computational time $\left(T_{\mathrm{c}}\right)$ in the harmonic-balanced methods depends on truncated harmonic number $\left(N_{\mathrm{c}}\right)$ and total number of iterations $\left(P_{\mathrm{c}}\right)$. Compared with the conventional HBFEM, the locally convergent fixed-point method converges fast and reduces the computational time significantly. 
COMPARISON OF THE TWO METHODS

\begin{tabular}{cccccc}
\hline \hline HB Method & $H \mathrm{~B}_{\mathrm{dcB}}(\mathrm{A} / \mathrm{m})$ & $U \mathrm{~B}_{\mathrm{acB}}(\mathrm{V})$ & $N \mathrm{~B}_{\mathrm{cB}}$ & $P \mathrm{~B}_{\mathrm{cB}}$ & $T \mathrm{~B}_{\mathrm{cB}}(\mathrm{s})$ \\
\hline Conventional & 107 & 290 & 9 & 53 & 3062.1 \\
Proposed & 107 & 290 & 9 & 26 & 1646.6 \\
Conventional & 213 & 420 & 11 & 74 & 8513.2 \\
Proposed & 213 & 420 & 11 & 18 & 2003.2 \\
\hline \hline
\end{tabular}

B. Discussion on Selection of Magnetization Curve in Computation of DC-biasing magnetic field

The basic magnetization curve is commonly used in computation of DC-biasing magnetic field [13]-[14]. In fact, the magnetization curve under DC-biased excitation differs from the basic one under sinusoidal excitation. Table I shows that the DC-biasing magnetization curve is not symmetric in the first and third quadrant of the $B-H$ coordinate. Therefore, the computational results obtained from the two curves must be different. Exciting current calculated from the two different magnetization curves are compared with the measured ones in Fig.7, and the corresponding quantitative comparison is presented in Table III. $I_{\mathrm{rms}}$ is the rms value of exciting current. It is obvious that the exciting current $i_{2}$ is more close to the measured result.

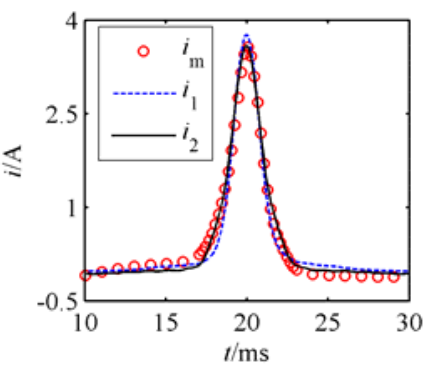

a) $U_{\text {ac }}=370 \mathrm{~V}$

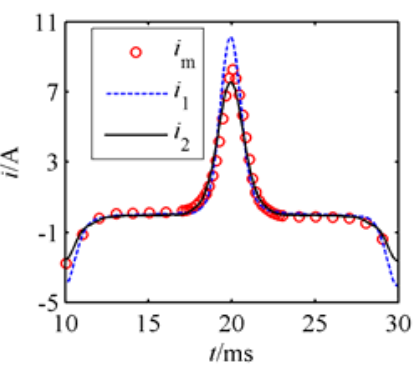

b) $U_{\text {ac }}=495 \mathrm{~V}$
Fig. 7. Comparison of calculated exciting currents $\left(H_{\mathrm{dc}}=107 \mathrm{~A} / \mathrm{m}\right) ; i_{1}$ and $i_{2}$ are exciting currents calculated by normal and DC-biasing magnetization curve respectively, and $i_{\mathrm{m}}$ is the measured result.

TABLE III

COMPARISON OF THE EXCITING CURRENT

\begin{tabular}{|c|c|c|c|}
\hline \hline$U_{\text {ac }}(\mathrm{V})$ & $I_{\text {rms,m }, \mathrm{m}}(\mathrm{A})$ & $I_{\mathrm{rms}, 1}(\mathrm{~A})$ & $I_{\mathrm{rms}, 2}(\mathrm{~A})$ \\
170 & 1.1264 & 1.1106 & 1.1536 \\
\hline 260 & 1.3511 & 1.3615 & 1.3841 \\
350 & 3.0379 & 3.6087 & 2.9125 \\
\hline \hline
\end{tabular}

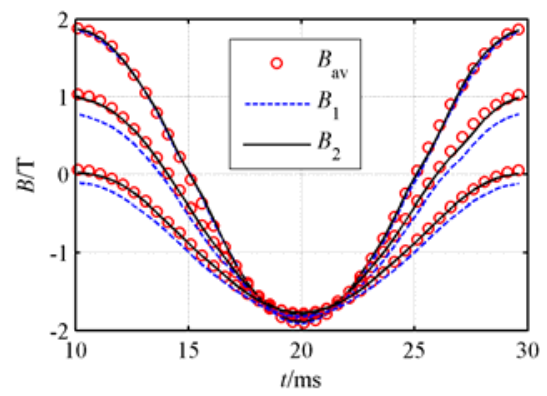

Fig. 8. Comparison of calculated flux density $\left(U_{\mathrm{ac}}=240 \mathrm{~V}, 370 \mathrm{~V}, 495 \mathrm{~V}\right.$; $\left.H_{\mathrm{dc}}=107 \mathrm{~A} / \mathrm{m}\right) . B_{1}$ and $B_{2}$ are flux density calculated by normal and DC-biasing magnetization curve respectively, $B_{\mathrm{av}}$ is the average flux density in (2).

TABLE IV

COMPARISON OF THE FLUX DENSITY

\begin{tabular}{ccccccc}
\hline \hline \multirow{2}{*}{$U_{\text {ac }}(\mathrm{V})$} & \multicolumn{2}{c}{$B_{\text {av }}(\mathrm{T})$} & \multicolumn{2}{c}{$B_{1}(\mathrm{~T})$} & \multicolumn{2}{c}{$B_{2}(\mathrm{~T})$} \\
& $B_{\mathrm{av}, \mathrm{dc}}$ & $B_{\mathrm{av}, \mathrm{ac}}$ & $B_{1, \mathrm{dc}}$ & $B_{1, \mathrm{ac}}$ & $B_{2, \mathrm{dc}}$ & $B_{2, \mathrm{ac}}$ \\
\hline 170 & -0.8582 & 0.9186 & -0.9727 & 0.8510 & -0.8681 & 0.8985 \\
260 & -0.3830 & 1.4163 & -0.5318 & 1.3056 & -0.4107 & 1.3865 \\
350 & -0.0100 & 1.8909 & -0.0330 & 1.8733 & -0.0072 & 1.8744 \\
\hline \hline
\end{tabular}

Point $E$ in SLC is selected to observe the waveforms of calculated flux density $\left(B_{1}\right.$ and $\left.B_{2}\right)$ under different DC-biased magnetization, and the computed results are compared with the measured one in Fig.8. $B_{\text {av }}$ is the sum of dc component $B_{\mathrm{av}, \mathrm{dc}}$ and ac component $B_{\mathrm{av}, \mathrm{ac}}$ related to $\varphi_{\mathrm{dc}}$ and $\varphi_{\mathrm{ac}}$ in (2) respectively. It is observed that the waveforms of flux density $B_{2}$ calculated by DC-biasing magnetization curve agrees better with that of the measured flux density $B_{\mathrm{av}}$. The numerical data is listed in detail in Table IV. Compared with $B_{1}$, the ac component $B_{2, \text { ac }}$ and dc component $B_{2, \mathrm{dc}}$ of $B_{2}$ coincide better with $B_{\mathrm{av}, \mathrm{ac}}$ and $B_{\mathrm{av}, \mathrm{dc}}$ respectively.

Based on the comparison between computed and measured results in terms of exciting current and flux density, the numerical results obtained from the DC-biasing magnetization curve are more accurate. It is indicated that the DC-biasing magnetization curve should be adopted in computation of magnetic field under DC-biased magnetization.

\section{CONCLUSION}

Unique features of hysteresis loops under DC-biased magnetization indicate that the characteristics of magnetization curve have changed when $\mathrm{dc}$ and ac flux coexist in laminated core. Asymmetric magnetization curve obtained from DC bias test is compared with the basic magnetization curve. The calculated results obtained from the former are more accurate than that obtained from the latter. The fixed-point technique is introduced in harmonic domain and combined with the HBFEM. The locally optimal scheme determines the fixed-point reluctivity in each iterative step to updates the coefficient matrix efficiently. The proposed method superior to the conventional algorithm shows excellent improvement in speeding up the convergence of harmonic solutions.

\section{REFERENCES}

[1] D. Miyagi, T. Yoshida and N. Takahashi, "Development of measuring equipment of dc-biased magnetic properties using open-type single-sheet tester," IEEE Trans. on Magn., vol.42, no.10, pp. 2846-2848, 2006.

[2] Z. Cheng, N. Takahashi, B. Forghani. Electromagnetic and Thermal Field Modeling and Application in Electrical Engineering, Beijing: Science Press, 2009, pp. 112-115.

[3] S. Ausserhofer, O. Biro and K. Preis, "An efficient harmonic balance method for nonlinear eddy-current problems," IEEE Trans. on Magn., vol.43, no.4, pp. 1229-1232, 2007.

[4] G. Koczka, S. Auberhofer, O. Biro and K. Preis, "Optimal convergence of the fixed-point method for nonlinear eddy current problems," IEEE Trans. on Magn., vol.45, no.3, pp. 948-951, 2009.

[5] S. Auberhofer, O. Biro and K. Preis, "A strategy to improve the convergence of the fixed-point method for nonlinear eddy current problems," IEEE Trans. on Magn., vol.44, no.6, pp. 1282-1285, 2008.

[6] E. Dlala, A. Belahcen and A. Arkkio, "Locally convergent fixed-point method for solving time-stepping nonlinear field problems," IEEE Trans. on Magn., vol.43, no.11, pp. 3969-3975, 2007.

[7] E. Dlala and A. Arkkio, "Analysis of the convergence of the fixed-point method used for solving nonlinear rotational magnetic field problems," IEEE Trans. on Magn., vol.44, no.4, pp. 473-478, 2008.

[8] Z. Zhao, F. Liu, S. L. Ho, W. N. Fu and W. Yan, "Modeling magnetic hysteresis under DC-Biased magnetization using the neural network," IEEE Trans. on Magn., vol.45, no.10, pp. 3958-3961, 2009.

[9] Z. Cheng, N. Takahashi, and B. Forghani, "Effect of variation of B-H properties on loss and flux inside silicon steel lamination," IEEE Trans. on Magn., vol.47, no.5, pp. 1346-1349, 2011. 
[10] F. I. Hantila, G. Preda and M. Vasiliu, "Polarization method for static field" IEEE Trans. on Magn., vol.36, no.4, pp. 672-675, 2000.

[11] S. Yamada, K. Bessho and J. Lu, "Harmonic balance finite element method applied to nonlinear AC magnetic analysis" IEEE Trans. on Magn., vol.25, no.4, pp. 2971-2973, 1989.

[12] X. Zhao, J. Lu, L. Li, Z. Cheng and T. Lu, "Analysis of the DC Bias phenomenon by the harmonic balance finite-element method," IEEE Trans. on Power Delivery., vol.26, no.1, pp. 475-485, 2010.

[13] Y. Yao, C. S. Koh, G. Ni and D. Xie, "3-D nonlinear transient eddy current calculation of online power transformer under dc bias," IEEE Trans. on Magn., vol.41, no.5, pp. 1840-1843, 2005.

[14] O. Biro, G. Buchgraber, G. Leber and K. Preis, "Prediction of magnetizing current wave-forms in a three-phase power transformer under dc bias," IEEE Trans. on Magn., vol.44, no.6, pp. 1554-1557, 2008 . 